\title{
Quantum-based Decision Making Under Uncertainty in the Presence of Entanglement for the Development of Optimal Strategies in Engineering Design
}

\author{
Evangelos Papageorgiou ${ }^{1}$, Eleni-Eleftheria Kamperi ${ }^{1}$ \\ ${ }^{1}$ Engineering Technology and Science, Department of Aviation Engineering, Higher Colleges of Technology, United \\ Arab Emirates \\ Correspondence: Evangelos Papageorgiou, Faculty Aviation Engineering and Technology, Higher Colleges of \\ Technology, ADMC, United Arab Emirates.
}

Received: February 17, 2017

Accepted: March 15, 2017 Online Published: March 17, 2017

doi:10.11114/set.v4i1.2280

URL: https://doi.org/10.11114/set.v4i1.2280

\begin{abstract}
The work presented concerns the development of a quantum-based decision making model utilised in the identification of optimal strategies in engineering design under uncertainty in a quantum-like entangled decision making, assessing intention interference. Several experiments and decision making paradoxes have manifested the deficiency of rationality assumption employed by classical decision making theory, with humans often violating the hypotheses of the expected utility theory and Game Theory by making irrational choices. Quantum Decision Theory (QDT) is the basis of the proposed decision making model, since only this theory can be employed to develop an operational tool in any social interplay, given the preferences of the individuals/players, to assess the quantum probabilities of their strategies. The decision makers' brain is assumed of a dual nature, with brain processes divided to conscious and subconscious constituent parts and the computed quantum strategic probabilities consisted of two parts, the first one being rationality related and the other one capturing biases, emotions and feelings. Hence, given a priori the preferences of the decision makers, this model can be utilised as an operational tool for decision making under uncertainty in the presence of entanglement assessing the quantum probabilities of the players' strategies in any engineering design. This quantum-based decision making model, identifying the optimal strategic choices of the stakeholders involved, is employed in the presented conceptual design of an Unmanned Air System (UAS), based on both the stakeholders' rationality, personal intuitive feelings and behavioural biases.
\end{abstract}

Keywords: Game Theory, Quantum Decision Theory (QDT), Quantum-based decision making model, Nash equilibrium, Nash Bargaining Solution (NBS), Unmanned Air System (UAS)

\section{Introduction}

In any decision making process, the identification and structuring of the decision maker's needs/requirements is the first step in the evaluation of the set of different decision alternatives. The goodness of any proposed solution is assessed with appropriate attributes, related to these needs and objectives, and is pronounced through the corresponding value model. The maximization of value is fundamental to any decision making problem solving and ideally, the value model employed in the human decision making process asserts the rational choice of maximum value as the optimal alternative.

Expected utility, multi-attribute utility theory, (Fishburn 1970), (Keeney and Raiffa 1976) and Game Theory (Von Neumann and Morgenstern 1953) have been used to measure the preferences of consequences with one or more dimensions, capturing the single and multiple decision makers' attitude towards uncertainty. Game Theory as an optimisation tool, modelling decision interactions among rational players as non-cooperative games, has been applied in numerous engineering design cases, as presented in Section 2. However various experiments, such as those performed by Tversky and Kahneman (1981), (1986) leading them to a Nobel Prize in Economic Sciences, have demonstrated the violation of rationality employed by the aforementioned approaches in the selection of the optimal choice. It is imperative that modern decision support systems capture the complexity of human decision making related with risk attitude in the presence of ambiguity and uncertainty, capturing the aforementioned violation of rationality. 
As Yukalov and Sornette (2014) discuss human decision making should not rely simply on the rationality basis of utility theory but instead on the basic feature of any living creature's intelligence for optimal decision making which is the adaptation to the environment. These decisions are an interaction between the conscious and subconscious brain processes. The former can be modelled by the deterministic utility theory, whilst the latter brain process, addressing biases, emotions and feelings, exhibits a rather stochastic behaviour that varies not only from one subject to another, but also for the same subject in different decisions and timeframes.

Recently, the mathematical models and concepts of quantum physics were applied in the formulation of uncertainty in human decision making to address the demonstrated violations of rationality. The application of quantum theory's mathematical modelling to human decision making and cognition does not necessarily imply that the human brain is considered to have a quantum nature. Consequently, these approaches are usually referred to as quantum-like instead of quantum mechanical or quantum, (Ashtiani and Azgomi 2015).

In this paper, we present a novel quantum-based model that can be employed in any non-cooperative complete information social game, such as the engineering design, used for the identification of the optimal strategies based on the players' preferences. This model captures successfully not just the rationality but also the subconscious intuitive feelings and emotions of the decision makers. The proposed 2-players game, based on Quantum Decision Theory (Yukalov and Sornette 2011), takes into account the dual nature, i.e. conscious and subconscious, of human decision making. Although most quantum-like methods follow a post-hoc approach to justify experimental findings and deviations from rationality, this model, given a priori the preferences of the decision makers as players, can be utilised as an operational tool to make decisions under uncertainty in the presence of entanglement. The assessed quantum strategic probabilities are then employed in the process of human decision making. Literature review concerning the application of Game Theory in any social game, such as engineering design, and the new approach of quantum-like decision making covering the deficiency of rationality assumption of classical decision making is presented in Section 2. The Quantum-based decision making model, as opposed to the classical decision making model of the 2-players non-cooperative social game, is presented in Section 3. An application of this model in a case study of engineering design, namely the selection of stakeholders' optimal strategies in a UAS conceptual design, is described in Section 4. Finally, primary contributions to the current state of knowledge and conclusions are outlined in Section 5.

\section{Background}

\subsection{Game Theory in Engineering Decision Making}

In all social sciences, such as economics, finance, sociology, and psychology, as well as engineering, several decisions made by different decision makers can be studied using the Game Theory. In general, all $N(>1)$ players aim to better promote their interests, through the maximization of their own particular objective function, while they are affected by the others' choices.

Although John Von Neumann is considered to be the founder of the Theory of Games (Von Neumann and Morgenstern 1953), the keystone was set by John Nash with his proposed Nash equilibrium solution of non-cooperative games (Nash 1950) and the Pareto optimal Nash bargaining solution (NBS) of cooperative games (Nash 1950), leading him to a Nobel Memorial Prize in Economic Sciences. The Nash equilibrium constitutes the set of all players' strategic choices and their corresponding payoffs, if each player has chosen a strategy and no player can benefit by changing their strategy, while the other players keep theirs unchanged. Nash equilibrium was employed in many engineering design cases, for example in the engineering asset management between maintenance chain participants in a negotiation model (Trappey, Trappey and Ni 2013), between engineering disciplinary teams for collaborative decision making (Xiao et al. 2005), and the design of an aero-structural aircraft wing shape optimisation (Desideri 2012).

The very common situation of indeterminacy arises in non-cooperative games, when these games have either none or multiple (pure) Nash equilibriums. In cases of multiple Nash equilibriums, Nash's product of the payoff/utility functions, proposed by Harsanyi (1995), could be utilised as the sole criterion for the selection of the single Nash equilibrium solution of the non-cooperative game. However, in general this indeterminacy can be defeated by allowing the players to play randomly using the Mixed Strategy Nash Equilibrium (MSNE). The randomisation of the pure strategies is employed for two reasons, first when there is not one pure strategy to be selected and second to confuse the other player. The indifference of the players between the strategic choices is used in the determination of the MSNE, i.e. the computation of the corresponding probabilities is done by equating the expected utilities of the player's strategies, (Hargreaves Heap and Varoufakis 2004). MSNE was employed for example in the design of tradable credit schemes on networks (He, Yin and Shirmohammadi 2013), in defining transport equilibrium (Dixit and Denant-Boemont 2014) and the design and analysis of auction-driven dynamic spectrum access in cognitive radio networks (Zhong et al. 2014).

Nash equilibrium of games between non-cooperative players does not guarantee the property of Pareto Optimality of the solution obtained. However, if players cooperate through a bargaining process, they will be rewarded with the 
Pareto optimal Nash bargaining solution (NBS), based on the criterion of maximization of the product of utilities' distances from the disagreement points, Nash (1950). Harsanyi and Selten (1972) generalized the bargaining problem for two or more players of not equal relative authorities. NBS has been identified in several cases modelled by cooperative games such as the design and management of microwave access networks (Jiao et al. 2011), design of controllers in a multi-agent team cooperation approach (Semsar-Kazerooni and Khorasani 2009), and collaborative product development (Arsenyan, Buyukozkan and Feyzioglu 2015).

\subsection{Quantum Theory and Decision Making}

Quantum Theory also known as quantum mechanics and quantum physics gradually arose first from Max Planck who introduced in the 1900's the solution to the black body radiation problem and the Albert Einstein's 1905 paper explaining the photoelectric effect, (Jammer 1966). Niels Bohr in the 1910's developed the theory of the atomic structure and the emanating radiation. Many great scientists and physicists such as Max Planck, Niels Bohr, Werner Heisenberg, Louis de Broglie, Arthur Compton, Albert Einstein, Erwin Schrödinger, Max Born, John von Neumann, Paul Dirac, Enrico Fermi, Wolfgang Pauli, Max von Laue, David Hilbert and others set the foundations of quantum mechanics, with several of them been awarded with the Nobel Prize for their contributions.

Although, quantum theory managed to explain and describe the atom better than classical mechanics and electromagnetism, humans and social groups as complex systems too, not only have a large number of unobservable states but also are extremely prone to being disturbed by measurements taken. Hence, following the brief introduction given by Ashtiani and Azgomi (2015) and Busemeyer (2009) presented in (Bruza et al. 2009), concerning the understanding of behavioural measures based on quantum probability principles, quantum theory could be employed as an alternative mathematical method for generating probability models.

Several observed decision making paradoxes and fallacies prove that classical decision making theory is incapable of effectively capturing human decision making, based on choices prescribed on the rationality basis of utility theory, Yukalov and Sornette (2014). A typical decision making paradox is the disjunction effect, i.e. the violation of the "sure-thing principle" formulated by Savage (1954): if alternative $A$ is preferred to alternative $B$, when event $X_{I}$ occurs and $A$ is also preferred to $B$, when event $X_{2}$ occurs, then $A$ should be preferred to $B$, when either $X_{1}$ or $X_{2}$ occur. The disjunction effect as a psychological phenomenon is the violation of the following total probability law:

$$
P\left(A \mid\left(X_{1} \cup X_{2}\right)\right)=P\left(X_{1}\right) \cdot P\left(\frac{A}{X_{1}}\right)+P\left(X_{2}\right) \cdot P\left(A / X_{2}\right)
$$

An example of the disjunction effect, presented in (Tversky and Shafir 1992), concerns the two-step gamble setup. A group of people could gamble and could either win or lose a certain amount of money; then, they could play again for a second time. According to their experiments, the majority would accept the second gamble when they knew the result of the first one (either win or lose), but only a minority would gamble again if the outcome of the first gamble was unknown to them. This experiment manifested the inherent aversion of humans towards uncertainty when making a decision (not knowing the outcome of the first gamble in this example). Other well-known decision making paradoxes that cannot be addressed by the expected utility theory, are the Allais paradox, (Allais 1953), the conjunction fallacy (Tversky and Kahneman 1983) and the Ellsberg paradox (Ellsberg 1990).

Quantum-like decision-making has been used as a new approach to cover the deficiency of rationality assumption employed in classical decision-making. Ashtiani and Azgomi (2015) presented all proposed quantum-like decision making models and discussed about their post-hoc nature. Most of these models are capable of addressing certain classical decision making paradoxes and justifying their experimental findings and deviations from rationality; but they fail in capturing the behaviour of average decision makers in a decision-making framework. Only Quantum Decision Theory (QDT) of Yukalov and Sornette (2011) can be used as an operational tool to make decisions under certainty in the presence of entanglement, given a priori the preferences of the players/decision makers. Other well-developed quantum decision-making models include the models of Busemeyer, Wang and Townsend (2006), Agrawal and Shard (2013) and Khrennikov (2010). Busemeyer, Wang and Townsend (2006) presented a rather simple and easy to understand quantum dynamic model describing in a post-hoc nature the disjunction effect in the prisoner's dilemma game. The quantum decision making model of Agrawal and Shard (2013) was based on QDT and focused also on the explanation of the disjunction effect providing general kinds of operators for the quantum interference equations. In the rigorous mathematical model of Khrennikov (2010), quantum-like information is processed based on classical electromagnetic signals induced by joint activity of neurons, assuming quantum computer functioning of the human brain. All of these models have a close similarity with QDT, incorporating an interference term in their formulation to justify the total probability law violation.

QDT is the only quantum method that can be employed within a general decision making framework not only to capture the decision makers' preferences through the classical utility theory, but also to address their beliefs, emotions and 
feelings through the interference term. This theory, presented by Yukalov and Sornette (2011), is based on the mathematical theory of complex separable Hilbert spaces through the superposition of composite prospects. It does not assume quantum properties of the brain; however it uses quantum techniques to generalize classical probability theory and address entangled decision-making, non-commutative subsequent decisions and intention interference.

In QDT, the probabilities of choosing different alternative actions are evaluated both consciously and subconsciously based on their usefulness and appeal/attraction to the decision maker. Very briefly, the basic aspects of QDT as presented in (Yukalov and Sornette 2014) are the following:

The closed linear envelope of the set of elementary prospects represented by vectors $|n\rangle$, composing the space of mind, is:

$$
\mathcal{H} \equiv \operatorname{Span}\{|n\rangle\}
$$

The prospects $\pi_{j}$ from the given set $\mathcal{L}$ are represented by the vectors $\left|\pi_{j}\right\rangle$ in the mind space. Then the prospect operators, which correspond to the local observables' operators in quantum theory, are defined as:

$$
\hat{P}\left(\pi_{j}\right) \equiv\left|\pi_{j}\right\rangle\left\langle\pi_{j}\right|
$$

The state of the decision maker is depicted by the non-negative operator $\rho$ acting on the space of mind and normalized, $\operatorname{Tr} \hat{\rho}=1$, with the trace taken over the mind space. The decision-maker's state is not defined by a simple wave function but by a statistical operator to allow influences by their environment. The probabilities of realizing any prospect are computed, representing the probability a given decision maker will choose this prospect given their strategic state of mind. These predicted probabilities are in general different for different subjects and for the same subject in different times. The prospect probabilities, playing the role of observable quantities are the averages of the prospect operators:

$$
p\left(\pi_{j}\right) \equiv \operatorname{Tr} \hat{\rho} \hat{P}\left(\pi_{j}\right)
$$

Writing down the explicit expression and separating diagonal and off-diagonal terms, the prospect probability in QDT is written in the following form:

$$
p\left(\pi_{j}\right)=f\left(\pi_{j}\right)+q\left(\pi_{j}\right)
$$

The first term represents the diagonal terms, called utility factor. Corresponding to a classical probability, it is an objective quantity reflecting the given properties of the prospect and its usefulness for the decision maker. The second term representing the off-diagonal terms, is the prospect's quantum interference term, called attraction factor and describing the prospect's attraction to the decision maker.

It is shown in (Yukalov and Sornette 2015) that the utility factor as a normalized function of prospect expected utility is:

$$
f\left(\pi_{j}\right)=\frac{U\left(\pi_{j}\right)}{\sum_{j} U\left(\pi_{j}\right) \exp \left[\beta U\left(\pi_{j}\right)\right]} \exp \left[\beta U\left(\pi_{j}\right)\right]
$$

The term $U\left(\pi_{j}\right)$ is the classical utility term of the prospect $\pi_{j}$ and $\beta$ is a confidence or certainty parameter. If no confidence is assumed then $\beta=0$, and the expression is reduced to:

$$
f\left(\pi_{j}\right)=\frac{U\left(\pi_{j}\right)}{\sum_{j} U\left(\pi_{j}\right)}
$$

The attraction factor, playing the role of hidden variables, incorporates subjective biases, emotions and feelings of the decision makers. Based on theorem 4.1 of (Yukalov and Sornette 2015), if the prospects and the decision makers' state $\hat{\rho}$ are entangled, then the attraction factors are non-zero. In QDT, the attraction factors have the following properties:

- The sum of all attraction factors is zero: $\sum_{j=1}^{N} q\left(\pi_{j}\right)=0$.

- They lie in the interval $[-1,1]$, with negative and positive values representing repulsive and attractive prospects, respectively.

It is shown in (Yukalov and Sornette 2011), that over many decision makers the attraction factor satisfies the quarter law: 


$$
\frac{1}{L} \sum_{j=1}^{L}\left|q\left(\pi_{j}\right)\right|=\frac{1}{4}
$$

Additionally, for decision makers exchanging information as members of society, the attraction factor diminishes in a decoherence process, (Yukalov and Sornette 2015).

Using the quarter law, the attraction factor for each prospect is assigned an average absolute value of $1 / 4$. Concerning the sign assignment of the attraction factor:

- $\quad$ Based on the well-documented assumption that most humans hesitate to act under uncertainty for decision making between acting or not acting under uncertainty, the non-acting prospect is always assumed more attractive and assigned a positive attraction factor of $1 / 4$, while the less attractive acting prospect is assigned a negative attraction factor of $-1 / 4$.

- For decision making under uncertainty between two different alternative prospects, the prospect providing a more certain gain (or more uncertain loss) is assumed more attractive than another prospect with less certain gain (less uncertain loss). In (Yukalov and Sornette 2014) the attractiveness of a prospect is characterized by how much the gain prevails over risk. Thus, for each prospect the ratio of maximal gain over the probability of minimal gain: $\frac{x_{\max }}{p\left(x_{\min }\right)}$ is used to compare the prospects in terms of their attractiveness.

Based on the QDT analysis, a prospect with a higher utility factor than another is considered more useful, with a higher attraction factor than another, more attractive, and with a higher prospect probability than another, more preferable. Thus, the optimal alternative prospect is the one with maximum probability among all prospects:

$$
p\left(\pi_{*}\right)=\max _{j} p\left(\pi_{j}\right),\left(\pi_{j} \in \text { Prospect Set } \mathcal{L}\right)
$$

The application of QDT within a decision making framework does not require the knowledge of quantum theory apart from some basic knowledge of Hilbert space analysis, since only the final expressions (5) - (9) are employed in the evaluation of the quantum prospect probabilities.

\section{Decision Making Model in a 2-Players Non-cooperative Social Game}

Game Theory studies any social interplay between $N(>1)$ individuals considered as stakeholders with different interests/stakes, who are all influenced by and influence the interests of all players. In this social game, each player seeks to better promote their own interests, through the maximization of their corresponding utility function $U_{i}, i=$ $1, \ldots, N$. The social game is assumed a game of complete information, with the players knowing each other's utility functions. The basic assumptions of Game Theory for these stakeholders as players of the game (Hargreaves Heap and Varoufakis 2004), are the following:

- The players are instrumentally rational, i.e. they act only according to their preferences as modelled by their objective functions.

- They share Common Knowledge of Rationality (CKR); an endless chain of beliefs, concerning their rationality, is created: each player is rational ( $0^{\text {th }}$ order of rationality), each player knows that each player is rational ( $1^{\text {st }}$ order of rationality), each player knows that each player knows that each player is rational ( $2^{\text {nd }}$ order) and so on.

- They have Common Priors or Consistently Aligned Beliefs (CAB), i.e. given the same information the rational players should draw the same conclusions.

- They share common knowledge of the game rules, i.e. they know all possible alternatives/acts of the game and the utility functions of all players. It is therefore, a game of perfect/complete information.

In the pursuit of values articulated by the stakeholders' objectives in the 2-players non-cooperative social game, each of them is forced to select particular strategies and make decisions, influencing the delivery of value to all others. Each stakeholder's objectives are modelled by the corresponding objective/utility function and the decisions made are considered as strategies, promoting these interests through the maximization of this objective function.

\subsection{Classical Decision Making Model in a 2-Players Non-cooperative Social Game}

The strategic choices of the stakeholders define the non-cooperative social game to be played, based on all possible combinations between the strategic decisions made by them. For a 2-players non-cooperative social game, the selection 
of the specific strategic choice for any of the two stakeholders depends not only on the value of their payoff /utility function but also on the expectations the player has concerning the other player's most likely strategy.

In Table 1, the general 2-players non-cooperative social game is presented with the payoff values shown for all combinations of strategies. In each cell of the table, the values of the players' utility/payoff functions are introduced. The + and - signs represent the best move for each player in response to each move of the other player. For example, the + sign next to the value of 0.5 of the Player 1's payoff function means that Strategy 2 is the best choice (as opposed to 0.4), if Player 2 selects Strategy 1. In a similar manner, the - sign next to the value of 0.7 of Player 2's payoff function means that Strategy 1 is the best response of Player 2 (as opposed to 0.5), if Player 1 selects Strategy 1. The cell that includes both + and - signs constitutes a Nash equilibrium and a potential solution of the game, since it represents the optimal strategic choice of both players. This selection does not maximize the objective function of each individual player (user and manufacturer), but represents the optimal strategic choice in response to the other player's strategic choice. In this case, through successive elimination of the strictly dominated strategies and based on the achieved values of payoff functions, Player 2 will always choose Strategy 1 from Strategy 2 as a strictly dominating strategy, irrespectively of what the other player does. Hence, Player 1 knowing this fact will select Strategy 2.

Table 1. General 2-Player Non-cooperative Game

\section{Players' Strategies \\ Player 2 Strategy 1 \\ Player 2 Strategy 2}

Player1 Payoff: 0.4

\section{Player 1 \\ Strategy 1}

Player1

Strategy 2
Player 2 Payoff: 0.7

Player 1 Payoff: 0.5

Player 2 Payoff: 0.8

Player 1 Payoff: 0.5

Player 2 Payoff: 0.5

Player 1 Payoff: 0.4

Nash

Player 2 Payoff: 0.6

If indeterminacy arises and multiple Nash equilibria are obtained in the game, Nash's product of the payoff/utility functions, proposed by Harsanyi (1995), can be utilised as the sole criterion for the selection of the specific Nash equilibrium solution of the non-cooperative game. Alternatively, the players are allowed to play randomly using the Mixed Strategy Nash Equilibrium (MSNE). The indifference of the players between their strategic choices is used as the basis for the computation of the corresponding strategies' probabilities, (Hargreaves Heap and Varoufakis 2004). In this case, the pure strategy 1 of player 2 strictly dominates strategy 2 and the MSNE are expressed as both probabilities of player 2 selecting strategy 1 and player 1 selecting strategy 2 equal to one.

\subsection{Quantum-based Decision Making Model in a 2-Players Non-cooperative Social Game}

As already discussed, the basic assumptions of Game Theory concerning the players of the game are, that they are instrumentally rational, they share Common Knowledge of Rationality (CKR), they have Common Priors or Consistently Aligned Beliefs (CAB) and they share common knowledge of the game rules (Hargreaves Heap and Varoufakis 2004). However, experiments as those performed by Tversky and Kahneman (1981), (1986) have demonstrated the violation of rationality, employed by the classical Game Theory in the selection of the optimal choice. Several empirical studies, commonly known as decision making paradoxes and fallacies, have manifested this deviation from utility and expected utility theory, (Fishburn 1970) and (Keeney and Raiffa 1976), respectively.

In the formulation of the classical decision making model, Game Theory successfully employs the expected utility theory as the only acceptable exemplar of rational behaviour; and it is used in this context to address the preferences of more than one stakeholder. The proposed quantum based decision making model allows capturing the complexity of human decision making related with risk attitude in the presence of ambiguity and uncertainty by incorporating apart from rationality, the decision makers' different biases, emotions and subjective feelings. Based on QDT, this model takes into account the dual nature, i.e. conscious and subconscious, of human decision making, and exhibits a stochastic behaviour through the assessment of quantum strategic probabilities.

Following the development of QDT presented by Yukalov and Sornette (2011), in the 2-players game two strategic choices/intentions one for each player are considered, each consisted of two strategies/representations. The first player's intention has the strategies / representations $\left|S_{1}\right\rangle$ and $\left|S_{2}\right\rangle$ and the second player's intention, the representations $\left|S_{1}{ }^{\prime}\right\rangle$ and $\left|S_{2}{ }^{\prime}\right\rangle$. Then, the two intention states for the first player are the following (and similarly for the second player), with coefficients defining the weights corresponding to the intended strategies, among which the choice is to be made:

$$
\left|\pi_{S_{1}}\right\rangle=a_{1}\left|S_{1} S_{1}{ }^{\prime}\right\rangle+a_{2}\left|S_{1} S_{2}{ }^{\prime}\right\rangle\left|\pi_{S_{2}}\right\rangle=b_{1}\left|S_{2} S_{1}{ }^{\prime}\right\rangle+b_{2}\left|S_{2} S_{2}{ }^{\prime}\right\rangle
$$


The general prospect state is the following:

$$
|\pi\rangle=c_{11}\left|S_{1} S_{1}{ }^{\prime}\right\rangle+c_{12}\left|S_{1} S_{2}{ }^{\prime}\right\rangle+c_{21}\left|S_{2} S_{1}{ }^{\prime}\right\rangle+c_{22}\left|S_{2} S_{2}{ }^{\prime}\right\rangle
$$

The decision making process based on the above general prospect state is entangled, taking also into account the preferences of the decision makers. Hence, these decisions, involving composite prospects, introduce interference terms.

The probabilities of strategies $S_{1}$ and $S_{2}$ are the following, as in QDT (Yukalov and Sornette 2011):

$$
p\left(S_{1}\right)=\left|a_{1}^{*} c_{11}+a_{2}^{*} c_{12}\right|^{2} \quad p\left(S_{2}\right)=\left|b_{1}^{*} c_{21}+b_{2}^{*} c_{22}\right|^{2}
$$

With $*$ denoting the transpose conjugate. The partial probabilities (probabilities of the intersection of two representations) and interference terms are introduced:

$$
\begin{gathered}
p\left(S_{1} S_{1}{ }^{\prime}\right) \equiv\left|a_{1} c_{11}\right|^{2}, \quad p\left(S_{1} S_{2}{ }^{\prime}\right) \equiv\left|a_{2} c_{12}\right|^{2}, \quad p\left(S_{2} S_{1}{ }^{\prime}\right) \equiv\left|b_{1} c_{21}\right|^{2}, \\
p\left(S_{2} S_{2}{ }^{\prime}\right) \equiv\left|b_{2} c_{22}\right|^{2} \\
q\left(S_{1}\right) \equiv 2 \operatorname{Re}\left(a_{1}^{*} c_{11} a_{2} c_{12}^{*}\right), \quad q\left(S_{2}\right) \equiv 2 \operatorname{Re}\left(b_{1}^{*} c_{21} b_{2} c_{22}^{*}\right)
\end{gathered}
$$

The uncertainty factors are defined as:

$$
\varphi\left(S_{1}\right) \equiv \cos \left[\arg \left(a_{1}^{*} c_{11} a_{2} c_{12}^{*}\right)\right], \quad \varphi\left(S_{2}\right) \equiv \cos \left[\arg \left(b_{1}^{*} c_{21} b_{2} c_{22}^{*}\right)\right]
$$

Then, the interference terms can be rewritten as:

$$
q\left(S_{1}\right)=2 \varphi\left(S_{1}\right) \sqrt{p\left(S_{1} S_{1}{ }^{\prime}\right) p\left(S_{1} S_{2}{ }^{\prime}\right)}, \quad q\left(S_{2}\right)=2 \varphi\left(S_{2}\right) \sqrt{p\left(S_{2} S_{1}{ }^{\prime}\right) p\left(S_{2} S_{2}{ }^{\prime}\right)}
$$

Hence, carrying out from equations (12) and after substituting the above terms (equations (13), (16)), the probabilities of strategies $S_{1}$ and $S_{2}$ are the following [equations (34) of (Yukalov and Sornette 2011)]:

$$
p\left(S_{1}\right)=p\left(S_{1} S_{1}{ }^{\prime}\right)+p\left(S_{1} S_{2}{ }^{\prime}\right)+q\left(S_{1}\right), \quad p\left(S_{2}\right)=p\left(S_{2} S_{1}{ }^{\prime}\right)+p\left(S_{2} S_{2}{ }^{\prime}\right)+q\left(S_{2}\right)
$$

The first two terms in the above equations are replaced by the utility factors of QDT, written in terms of the classical utility terms according to equation (7) as:

$$
f\left(S_{1}\right)=\frac{U\left(S_{1}\right)}{U\left(S_{1}\right)+U\left(S_{2}\right)}, \quad f\left(S_{2}\right)=\frac{U\left(S_{2}\right)}{U\left(S_{1}\right)+U\left(S_{2}\right)}
$$

After computing the expected utility of each strategy for each player, the utility factors are obtained based on the above equations. Thus, following the assessment of the interference terms $q(S)$ presented in the next section, the probabilities of strategies $S_{1}$ and $S_{2}$ are computed as (and similarly for strategies $S_{1}{ }^{\prime}$ and $S_{2}{ }^{\prime}$ ):

$$
p\left(S_{1}\right)=f\left(S_{1}\right)+q\left(S_{1}\right), \quad p\left(S_{2}\right)=f\left(S_{2}\right)+q\left(S_{2}\right)
$$

\section{Attraction Criterion}

As already discussed, in QDT the sign assignment of the attraction factor is based on two attraction criteria: when deciding between acting and not acting under uncertainty, and when selecting under uncertainty between two different alternative prospects. The following can be argued concerning the attraction factor's sign assignment:

- The well-documented first criterion assumes that the non-acting prospect is more attractive to most humans when deciding under uncertainty. This is not applicable within a general decision making framework, with the decision maker selecting between alternative strategies.

- Concerning the second criterion which is applicable in a general decision making framework, when comparing the attractiveness of two prospects (Yukalov and Sornette 2014), the following could be argued: This criterion is based on the assumption that the prospect providing a more certain gain is more attractive to humans than 
another prospect with less certain gain. The ratio of maximal gain over the probability of minimal gain: $\frac{x_{\max }}{p\left(x_{\min }\right)}$ for each alternative prospect was used in equation (51) of (Yukalov and Sornette 2014) to compare two prospects in terms of attractiveness. The bias captured with this criterion is the human attraction towards achieving maximal gain while at the same time minimizing the risk of having minimal gain. However, it fails to capture other human feelings such as reciprocity, mutual trust and altruism. The ratio of maximal gain over the probability of minimal gain for each alternative prospect could simply be considered as another mathematical expression, based on the individual's instrumental rationality when deciding under uncertainty, similar to the expected utility form of utility theory: $U=p\left(x_{\max }\right) \cdot x_{\max }+p\left(x_{\min }\right) \cdot x_{\min }$.

- Another similar criterion could be introduced to model the human risk aversion and attraction to the prospect that provides the largest certain (guaranteed) gain. The prospect that provides a higher value of the lowest possible gain $x_{\min }$ is considered more attractive than another with a lower value of $x_{\min }$. Based on the Maximin solution proposed by Von Neumann and Morgenstern (1953) to identify the best strategy in zero-sum games, the player is attracted to the prospect with the highest value of the worst gain among all possible gains of this prospect. This Maximin criterion ascertains that the player will always be warranted for the largest lowest gain.

- The irrational behaviour of the disjunction effect observed in the classical prisoner's dilemma games (Hargreaves Heap and Varoufakis 2004) and the public good game (Ledyard 1995) is undoubtedly related with biases, emotions and feelings, such as learning, reciprocity, mutual trust and altruism. QDT captures the effect of learning in a decoherence process by diminishing the attraction factor through information exchange (Yukalov and Sornette 2015). However, by using the above ratio of maximal gain over the probability of minimal gain: $\frac{x_{\max }}{p\left(x_{\min }\right)}$ for each alternative prospect, it fails to address reciprocity, mutual trust and altruism.

- To capture the tendency towards reciprocal or conditional cooperation between the players of a non-cooperative game, an alternative criterion, used in cooperative games to obtain the Nash Bargaining solution, is introduced. The cooperation of the players reaching a binding agreement is promoted by achieving a Pareto optimal solution through the maximization of the product of utilities' distances from their disagreement points. Hence, in the 2-player non-cooperative game, a strategy is deemed more attractive than another, if it better promotes cooperation between the players against the rationality of non-cooperation. This attractiveness is assessed through a cooperation criterion, measured with the product of utility values of the players for all combinations of strategies. This criterion capturing mutual trust and reciprocity between the players is in accordance with the assumption of Yukalov and Sornette (2014) who assume that cooperation is more attractive than defection in the prisoner's dilemma games.

Summarizing, the sign of the attraction factor for each strategy is defined based on the following three criteria:

1) The (Yukalov and Sornette 2014) proposed attraction ratio criterion, using the ratio of maximum utility value over the probability of minimum utility value: $\frac{U_{\max }}{p\left(U_{\min }\right)}$ for each alternative strategy.

2) The Maximin criterion, using the lowest utility value among all possible utilities of each strategy to describe attraction towards the largest guaranteed gain strategy.

3) The cooperation criterion, measured with the product of values of utilities of the two players for all combinations of strategies to address attraction to reciprocity and mutual trust.

In the proposed quantum based decision-making model, any combination of these attractions can be selected to superimpose multiple biases, emotions and feelings of the players, enforcing or cancelling out their individual effect. It is also possible to select different criteria for each player, i.e. for one player attracted to reciprocity and mutual trust use the cooperation criterion, while for the other risk averse player select the maximin criterion. As in Table 2, depending on the players' feelings, emotions and biases and the corresponding criteria, each player's strategy is assigned a +1 or -1 (i.e. attractive or repulsive), used in the computation of the corresponding values of the attraction factor for each player's strategy (initially set to $\pm 1 / 4$ ), presented in the next section. 
Table 2. Attraction Factor Sign Assignment

\begin{tabular}{llllll}
\hline $\begin{array}{l}\text { Multiple Criteria for sign } \\
\text { definition of Attraction } \\
\text { Factor }\end{array}$ & $\begin{array}{l}\text { Maximin } \\
\text { Criterion }\end{array}$ & $\begin{array}{l}\text { Cooperation } \\
\text { Criterion }\end{array}$ & $\begin{array}{l}\text { Attraction } \\
\text { Ratio Criterion }\end{array}$ & $\begin{array}{l}\text { Overall } \\
\text { Criterion }\end{array}$ & $\begin{array}{l}\text { Initial Values of } \\
\text { Attraction Factor }\end{array}$ \\
\hline $\begin{array}{l}\text { Player 1 } \\
\text { Criteria }\end{array}$ & Yes & Yes & Yes & \\
$\begin{array}{l}\text { Player 2 } \\
\text { Criteria }\end{array}$ & Yes & Yes & Yes & & \\
Player 1 & & & & -1 & -0.25 \\
Strategy 1 & 1 & -1 & -1 & 1 & 0.25 \\
Player 1 & & & & 1 & 0.25 \\
Strategy 2 & -1 & 1 & 1 & -1 & -0.25 \\
Player 2 Strategy 1 & 1 & 1 & 1 & -1 & \\
Player 2 Strategy 2 & -1 & -1 & & & \\
\hline
\end{tabular}

Quantum Strategic Probabilities Assessment

Concerning the interference term of equations (16), Yukalov and Sornette (2011) assume no other prior information and use the uniform distribution of the absolute values of the uncertainty factors in the interval $[0,1]$ to assign them an average absolute value of $1 / 2$. They also assume the non-informative prior for all probabilities in the square roots of equation (16) and their expected values all being 1/2. Substituting these values, the quarter law for the attraction factor is derived:

$$
\left|q\left(\pi_{A}\right)\right|=\left|q\left(\pi_{B}\right)\right|=\frac{1}{4}
$$

In the proposed quantum-based decision making model, the probabilities of the players selecting strategies $\left(S_{1}, S_{2}, S_{1}{ }^{\prime}\right.$ and $S_{2}{ }^{\prime}$ ) are initially set to $1 / 2$, as in QDT, and all attraction factors are initially assigned an absolute magnitude of $1 / 4$, following the quarter law. However, the quantum strategic probabilities and the attraction factors are computed according to the following process, also presented in Figure 1:

- Identify the players' biases, feelings and emotions and employ appropriate attraction criteria, as discussed in the previous section.

- Since no prior information is given, the expected values of the probabilities of the strategies $\left(S_{1}, S_{2}, S_{1}{ }^{\prime}\right.$ and $\left.S_{2}{ }^{\prime}\right)$ of the players are initially set to $1 / 2$, as in QDT. The initial values of the attraction factors are set to $1 / 4$, according to the quarter law.

- Next, the values of the expected utility functions of the players' strategies are computed: $U\left(S_{i}\right)=p\left(S_{1}{ }^{\prime}\right)$. $U\left(S_{i 1}\right)++p\left(S_{2}{ }^{\prime}\right) \cdot U\left(S_{i 2}\right)$, with $P\left(S_{1}^{\prime}\right)$ and $P\left(S_{2}^{\prime}\right)$ the probabilities that the other player will select strategies $S_{1}{ }^{\prime}, S_{2}{ }^{\prime}$ (initially set to $1 / 2$ ) and $U\left(S_{i 1}\right), U\left(S_{i 2}\right)$ the values of the utility functions of the player for strategy $S_{i}$ when the other player selects strategies $S_{1}{ }^{\prime}$ and $S_{2}{ }^{\prime}$ respectively.

- The utility factors are obtained from the expected utilities of the two strategies of each player, according to

equations (18), i.e.: $f\left(S_{1}\right)=\frac{U\left(S_{1}\right)}{U\left(S_{1}\right)+U\left(S_{2}\right)}, f\left(S_{2}\right)=\frac{U\left(S_{2}\right)}{U\left(S_{1}\right)+U\left(S_{2}\right)}$.

- The strategic probabilities of equations (19) are computed by adding the computed utility factors and the current values of the attraction factors: $p\left(S_{i}\right)=f\left(S_{i}\right)+q\left(S_{i}\right)$.

- The probabilities of joint strategies: $p\left(S_{1} S_{1}{ }^{\prime}\right), p\left(S_{1} S_{2}{ }^{\prime}\right), p\left(S_{2} S_{1}{ }^{\prime}\right), p\left(S_{2} S_{2}{ }^{\prime}\right)$ are calculated as: $p\left(S_{i} S_{j}{ }^{\prime}\right)=$ $p\left(S_{i} \mid S_{j}^{\prime}\right) \cdot p\left(S_{j}{ }^{\prime}\right)$. The probability $p\left(S_{i} \mid S_{j}^{\prime}\right)$ is computed, using the values of the utility function $U\left(S_{i j \prime}\right)$ and $U\left(S_{k j^{\prime}}\right)$ for strategies $S_{i}$ and $S_{k}$ of player 1 when strategy $S_{j}^{\prime}$ is selected by player 2 , as: $p\left(S_{i} \mid S_{j}^{\prime}\right)=$ $\frac{U\left(S_{i j \prime}\right)}{U\left(S_{i j \prime}\right)+U\left(S_{k j \prime}\right)}+q\left(S_{i}\right)$ and $q\left(S_{i}\right)$ the current value of the attraction factor of strategy $S_{i}$. Also, $p\left(S_{j}{ }^{\prime}\right)$ is the current value of the probability of player 2 selecting strategy $S_{j}^{\prime}$ (and similarly for player 2). 
- The attraction factors are calculated using equation (16) based on the values of joint strategies' probabilities, computed in the previous step. As in QDT, for a binary strategy set without any other information available, the uncertainty factor $\varphi\left(S_{1}\right)$ of equation (16), related to the existence of uncertainty in decision-making, is set to 0.5 . Then, the property of interference alteration: $\sum_{j=1}^{N} q\left(\pi_{j}\right)=0$ (i.e. equal in magnitude for binary strategy set) is used to assess the other uncertainty factor $\varphi\left(S_{2}\right)$.

- The above computed values of quantum strategic probabilities and attraction factors replace the previous values, and they are used to update the strategies' expected utilities, utility factors etc. The iteration process carries on until convergence is achieved between the previous values and the computed values of quantum strategic probabilities and attraction factors of strategies $\left(S_{1}, S_{2}, S_{1}{ }^{\prime}\right.$ and $\left.S_{2}{ }^{\prime}\right)$.

Thus, during this iteration, the values of quantum strategic probabilities and attraction factors of the players are updated and the final converged values reflect their most accurate assessment.

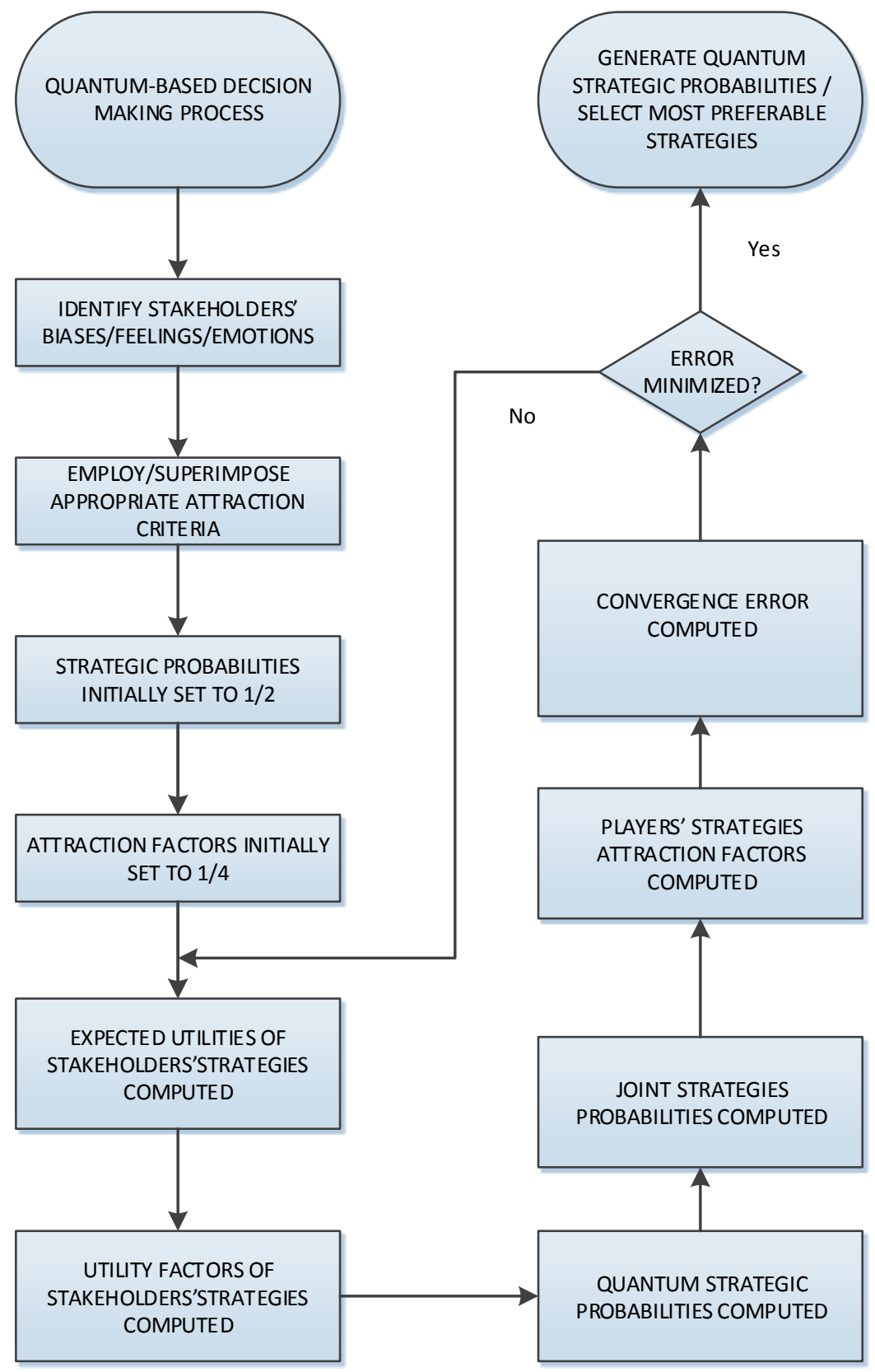

Figure 1. Quantum-based Strategic Probabilities and Attraction Factors Assessment

\section{Quantum-based Decision Making in Engineering Design}

To identify the value-enhancing design(s) in engineering design, the optimization process should explore the widest possible design space and address all economic and non-economic needs of stakeholders involved. Hence, the goodness 
of all proposed system solutions needs to be assessed through the use of multiple criteria decision analysis approach and Game Theory needs to be applied in a social game between all stakeholders involved as players. In (Papageorgiou, Eres and Scanlan 2015) value driven design philosophy was applied in the conceptual design of a small Unmanned Air System (UAS) utilised in defence applications to locate the best design depending on the user's needs/requirements. Furthermore, rational behaviour was assumed as the basis of a classical Game Theory application incorporating beyond the user's, the manufacturer's preferences also in this design in (Papageorgiou, Eres and Scanlan 2016). A hybrid cooperative/non-cooperative, non-zero sum game between the user and the manufacturer was developed to identify both the optimal UAS design and their optimal strategic choices through the simultaneous employment of Nash bargaining solution and Nash equilibrium.

Concerning the user's and manufacturer's available strategic choices, modelled in the 2-players, non-zero sum, non-cooperative game, the list includes different performance requirements such as cruise speed, improvement of technology, different quality control processes, and so on, assumed constant in the design space exploration. For demonstration purposes, in this 2-players game the UAS manufacturer has the option of selecting between two different levels of reliability of UAS components, one lower level of cheaper and less reliable components and one higher level of more reliable and more expensive components. The user selects between two different maintenance policies, one policy of replacing UAS components at scheduled time intervals (measured in flight hours) depending on their reliability, and one policy of simply replacing the whole UAS at scheduled times. Both strategic choices of the stakeholders have obviously a significant impact on aircraft acquisition cost, scheduled maintenance cost, total lifecycle cost and UAS losses during operations. In Table 3, this game is presented with the corresponding values of the two stakeholders' utility functions. These values of the stakeholders' multi-attribute utility functions reflect their personal preferences in a series of different objectives, from operational surveillance time, maximum endurance, maximum range to total lifecycle cost and defence related survivability and detectability of the UAS.

Table 3. User - Manufacturer Non-Cooperative Game

\begin{tabular}{|c|c|c|c|c|c|c|c|}
\hline $\begin{array}{l}\text { Manufacturer's } \\
\text { User's Strategies }\end{array}$ & & $\begin{array}{l}\text { Component } \\
\text { Policy }\end{array}$ & Replacement & Maintenance & & UAS I & ment Policy \\
\hline Original Reliability & + & 0.418 & 0.699 & $\begin{array}{l}\text { Nash } \\
\text { Equilibrium }\end{array}$ & + & 0.524 & 0.495 \\
\hline $\begin{array}{l}\text { Improved } \\
\text { Reliability }\end{array}$ & & 0.416 & 0.725 & $\begin{array}{l}\text { Nash } \\
\text { Bargaining } \\
\text { Solution }\end{array}$ & & 0.384 & 0.643 \\
\hline
\end{tabular}

In (Papageorgiou, Eres and Scanlan 2016), the selection of these alternative strategies was captured as a process of strategic interactions between the players of a non-cooperative game. The combination of the players' strategies that does not maximize the objective function of each individual player but represents the optimal strategic choice in response to the other player's strategic choice constitutes the Nash equilibrium, as indicated in Table 3. This Nash equilibrium defines the solution of the 2-players, pure non-cooperative, non-zero sum social game, justified through successive elimination of the strictly dominated strategies, (Nash 1950). Alternatively, this engineering design was also modelled as a fully cooperative, non-zero sum game, solved as a pure bargaining problem (Nash 1950); and the optimal solution was based solely on the criterion of the maximization of the product of their payoff functions, also indicated in Table 3.

The quantum-based decision making model, presented in section 0 , is employed in this engineering design with the two stakeholders as players of a 2-players social game. In this decision making model beyond rational behaviour, biases, emotions and intuitive feelings of the two stakeholders are modelled to assess the quantum strategic probabilities. Each of the two stakeholders has to make a decision between two alternative strategies based not only on the values of their personal utility functions, modelling their preferences, but also based on their own personal intuitive feelings and behavioural biases.

Quantum-based Engineering Design Methodology

The methodology of the proposed decision making process in the engineering design is the following, also presented in Figure 2:

- The stakeholders and their strategic choices are first identified and the quantum-based game is formed.

- The values of the stakeholders' utility functions for the different strategies are assessed, as in Table 3.

- The stakeholders' biases, emotions and feelings are identified and appropriate attraction criteria are employed and superimposed to obtain the overall attraction criterion, used in the sign definition of the attraction factor, as in Table 2. 
- The expected values of the probabilities of the stakeholders' strategies are initially set to $1 / 2$, as in QDT. The initial values of the attraction factors are set to $\frac{1}{4}$ or $-\frac{1}{4}$, based on the quarter law and the sign defined in the previous step.

- The expected utilities of each strategy/prospect for both stakeholders are obtained as: $U\left(S_{i}\right)=p\left(S_{1}^{\prime}\right)$. $U\left(S_{i 1}\right)+p\left(S_{2}^{\prime}\right) \cdot U\left(S_{i 2}\right)$, with $p\left(S_{1}^{\prime}\right)$ and $p\left(S_{2}^{\prime}\right)$ the probabilities that the other stakeholder will select strategies $S_{1}{ }^{\prime}, S_{2}{ }^{\prime}$ and $U\left(S_{i 1}\right), U\left(S_{i 2}\right)$ the values of the utility functions of the stakeholder for strategy $S_{i}$ when the other stakeholder selects strategies $S_{1}{ }^{\prime}$ and $S_{2}{ }^{\prime}$ respectively (from Table 3).

- The utility factors for each of the two stakeholders are computed from the expected utilities of the two strategies of each stakeholder, according to equations (18), i.e.: $f\left(S_{1}\right)=\frac{U\left(S_{1}\right)}{U\left(S_{1}\right)+U\left(S_{2}\right)}, f\left(S_{2}\right)=\frac{U\left(S_{2}\right)}{U\left(S_{1}\right)+U\left(S_{2}\right)}$.

- The quantum strategic probabilities of the strategies of the stakeholders are obtained, equation $(19): p\left(S_{i}\right)=$ $f\left(S_{i}\right)+q\left(S_{i}\right)$.

- The probabilities of joint strategies: $p\left(S_{1} S_{1}{ }^{\prime}\right), p\left(S_{1} S_{2}{ }^{\prime}\right), p\left(S_{2} S_{1}{ }^{\prime}\right), p\left(S_{2} S_{2}{ }^{\prime}\right)$ are calculated as: $p\left(S_{i} S_{j}{ }^{\prime}\right)=$ $p\left(S_{i} \mid S_{j}^{\prime}\right) \cdot p\left(S_{j}{ }^{\prime}\right)$, with $p\left(S_{i} \mid S_{j}^{\prime}\right)$ the probability of one stakeholder selecting strategy $S_{i}$ when the other selects strategy $S_{j}{ }^{\prime}$, and $p\left(S_{j}{ }^{\prime}\right)$ the probability of the other stakeholder selecting strategy $S_{j}{ }^{\prime}$ and computed as described in 0 .

- $\quad$ The attraction factors are calculated using equation (16).

- The convergence error is computed as the difference between the computed quantum strategic probabilities and attraction factors and their previous values.

- If the error is not less than the desired accuracy, the computed values of the quantum strategic probabilities and attraction factors are used to update the strategies' probabilities and expected utilities and the iteration process carries on until convergence is achieved.

- The final converged values of the quantum strategic probabilities are obtained for all strategies.

- The stakeholders' optimal strategies are identified as those strategies maximizing the values of quantum strategic probabilities. 


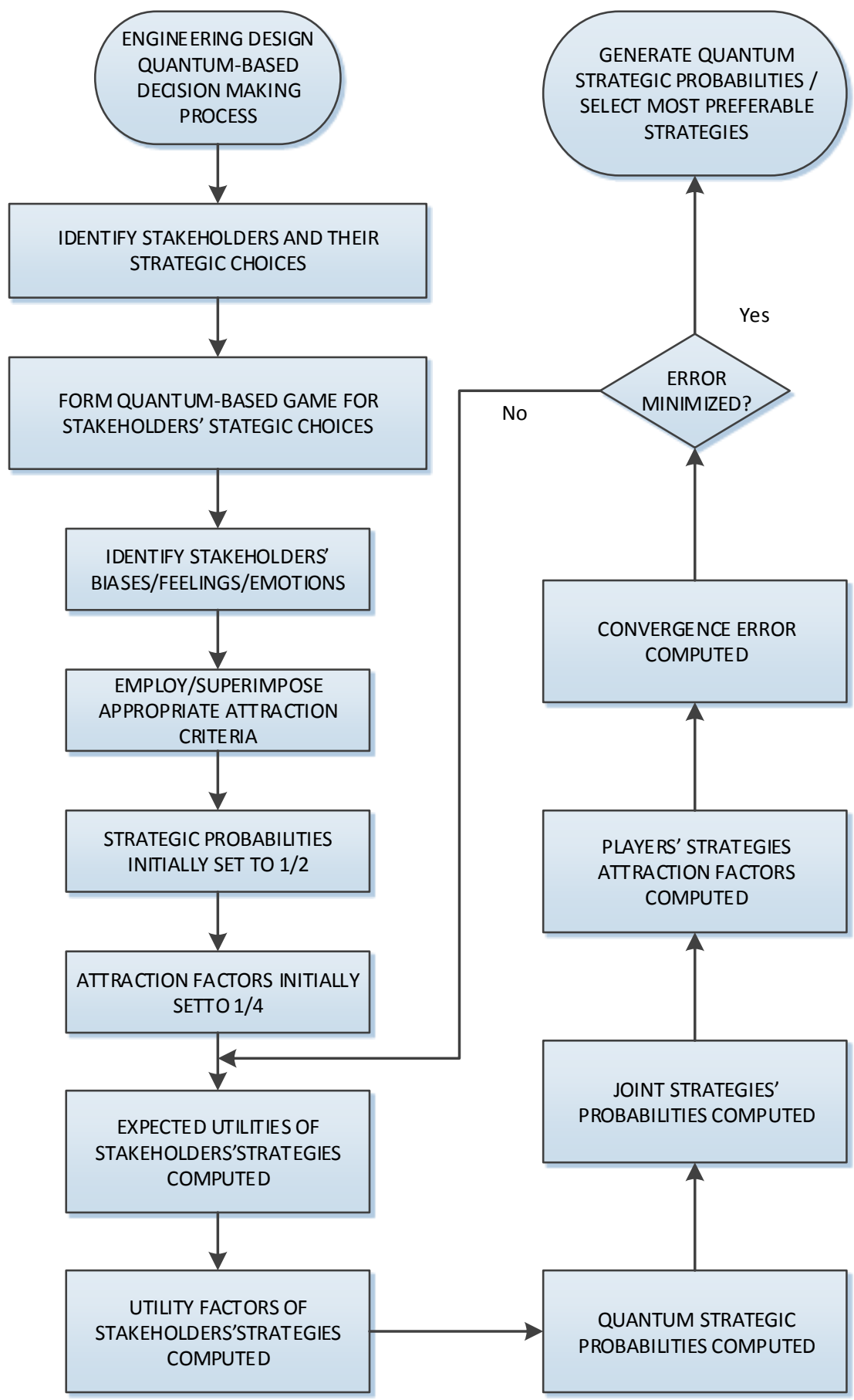

Figure 2. Engineering Design Quantum-based Decision Making Process

For the UAS conceptual design, the final converged values of the quantum strategic probabilities are presented in Table 4. The attractiveness of the strategies was defined based on the attraction ratio criterion, the maximin criterion and the cooperation criterion. Only the values of the attraction factors of the original reliability and UAS component replacement policy strategies are presented, since the other strategies' attraction factors take the opposite value. With the maximin criterion capturing the attraction to the prospect that provides the largest guaranteed gain, the strategy that had larger lower utility than the other strategy was considered more attractive. Using the attraction ratio criterion, the ratio of maximum value of utility (between $U\left(S_{i 1}\right)$ and $U\left(S_{i 2}\right)$ ) over the corresponding probability of minimum value of utility, $\frac{U_{\max }}{p\left(U_{\min }\right)}$ was used compare the two alternative strategies in terms of attractiveness. To capture the tendency 
towards reciprocal cooperation between the stakeholders, the strategies' attractiveness was assessed with the cooperation criterion and was measured with the product of utility values of the players for all combinations of strategies. In the fourth column, the cooperation and maximin criteria were selected to superimpose the stakeholders' attractions towards reciprocity, mutual trust and risk aversion, and finally all criteria were superimposed in the fifth column.

Table 4. Quantum-based Decision Making Engineering Design

\begin{tabular}{|c|c|c|c|c|c|c|}
\hline $\begin{array}{l}\text { Quantum-basec } \\
\text { Making } \\
\text { Design }\end{array}$ & $\begin{array}{l}\text { Decision } \\
\text { Engineering }\end{array}$ & $\begin{array}{l}\text { Maximin } \\
\text { Criterion }\end{array}$ & $\begin{array}{l}\text { Cooperation } \\
\text { Criterion }\end{array}$ & $\begin{array}{l}\text { Attraction } \\
\text { Ratio } \\
\text { Criterion }\end{array}$ & $\begin{array}{l}\text { Cooperation - } \\
\text { Maximin } \\
\text { Criteria } \\
\text { Superimposed }\end{array}$ & $\begin{array}{l}\text { All Criteria } \\
\text { Superimposed }\end{array}$ \\
\hline & $\begin{array}{l}\text { Original } \\
\text { Reliability }\end{array}$ & 0.668 & 0.356 & 0.356 & 0.525 & 0.356 \\
\hline & $\begin{array}{l}\text { Improved } \\
\text { Reliability }\end{array}$ & 0.332 & 0.644 & 0.644 & 0.475 & 0.644 \\
\hline \multirow[t]{4}{*}{$\begin{array}{l}\text { Players' } \\
\text { Quantum } \\
\text { Probabilities }\end{array}$} & $\begin{array}{l}\text { Component } \\
\text { Replacement } \\
\text { Maintenance } \\
\text { Policy }\end{array}$ & 0.707 & 0.691 & 0.691 & 0.704 & 0.691 \\
\hline & $\begin{array}{l}\text { UAS } \\
\text { Replacement } \\
\text { Policy }\end{array}$ & 0.293 & 0.309 & 0.309 & 0.296 & 0.309 \\
\hline & $\begin{array}{l}\text { Original } \\
\text { Reliability }\end{array}$ & 0.525 & 0.526 & 0.526 & 0.525 & 0.526 \\
\hline & $\begin{array}{l}\text { Improved } \\
\text { Reliability }\end{array}$ & 0.475 & 0.474 & 0.474 & 0.475 & 0.474 \\
\hline \multirow[t]{3}{*}{$\begin{array}{l}\text { Utility } \\
\text { Factors }\end{array}$} & $\begin{array}{l}\text { Component } \\
\text { Replacement } \\
\text { Maintenance } \\
\text { Policy }\end{array}$ & 0.565 & 0.548 & 0.548 & 0.557 & 0.548 \\
\hline & $\begin{array}{l}\text { UAS } \\
\text { Replacement } \\
\text { Policy }\end{array}$ & 0.435 & 0.452 & 0.452 & 0.443 & 0.452 \\
\hline & $\begin{array}{l}\text { Original } \\
\text { Reliability }\end{array}$ & 0.144 & -0.169 & -0.169 & 0 & -0.169 \\
\hline $\begin{array}{l}\text { Attraction } \\
\text { Factors }\end{array}$ & $\begin{array}{l}\text { Component } \\
\text { Replacement } \\
\text { Maintenance } \\
\text { Policy }\end{array}$ & 0.141 & 0.143 & 0.143 & 0.147 & 0.143 \\
\hline
\end{tabular}

From the results of Table 4, the following are observed: The strategy of original reliability dominates the strategy of improved reliability in terms of usefulness. However, its attraction factor varies from -0.169 to 0 and 0.144 , depending on which attractiveness criterion is used. This strategy is the most attractive for the manufacturer, using the maximin criterion, since it provides a higher lowest utility value than the improved reliability strategy (0.418 vs. 0.384$)$; and consequently it is the most preferable. However, when using either the cooperation attractiveness criterion or the attraction ratio criterion or superimposing all criteria, the improved reliability strategy appears as the most preferable choice, since it is the most attractive for the manufacturer. With the fourth option of selecting both the cooperation and maximin criteria, none of the two levels of reliability is attractive to the manufacturer and only usefulness (i.e. rationality) is employed to identify the strategy of original reliability as the most preferable. Due to the obtained values of the user's utility function, the strategy of UAS component replacement maintenance policy is strictly dominating the UAS replacement policy, irrespective what the manufacturer selects; and as expected, it is found more useful. However, it is always more preferable because it is more attractive than the UAS replacement policy using all different attractiveness criteria or combinations of them. 
Table 5. UAS Design Stakeholders' Optimal Strategies

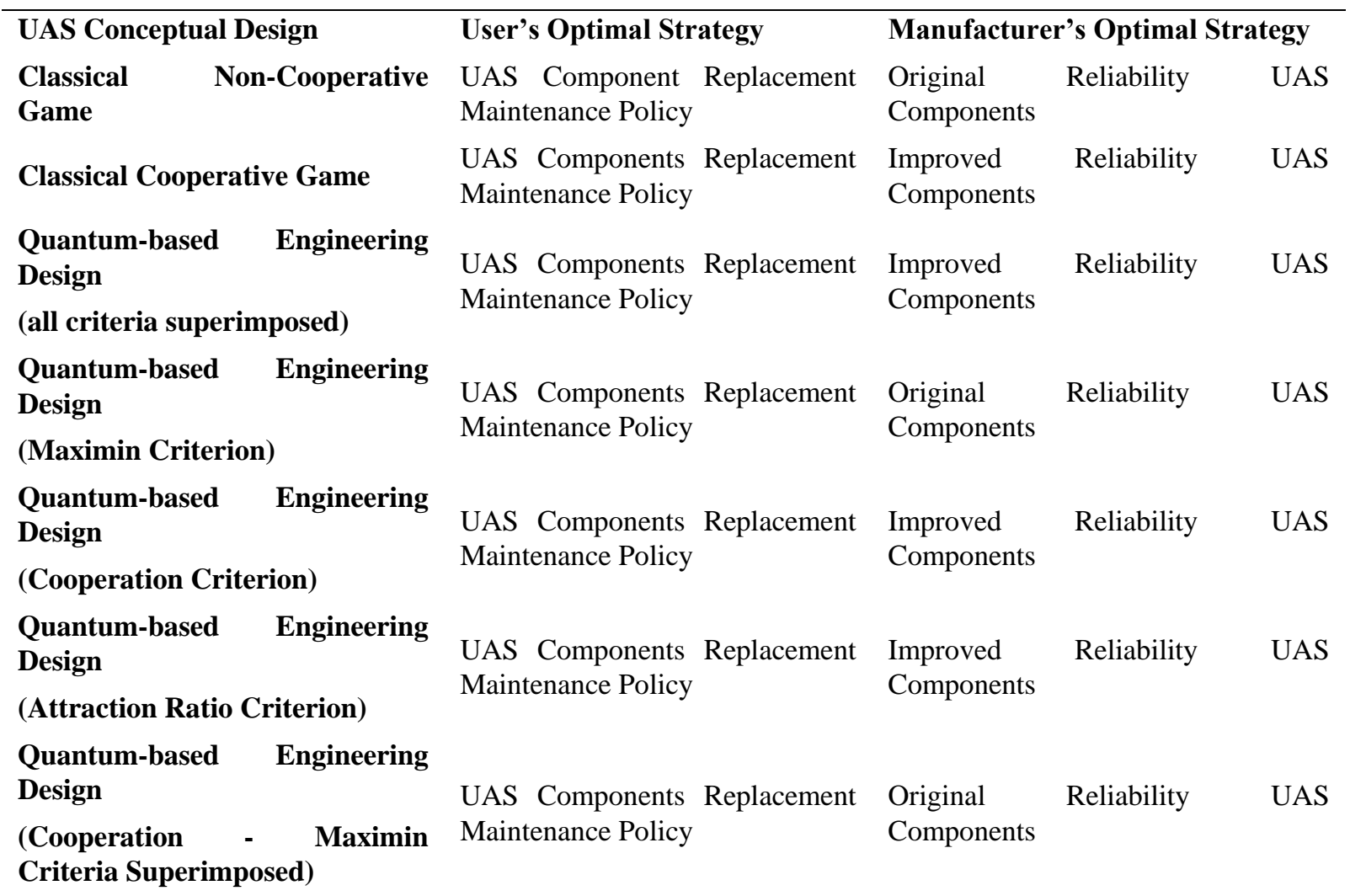

Summarizing, as presented also in Table 5, the non-cooperative game identified the manufacturer's strategy of original level of reliability for UAS components and the user's strategy of UAS component replacement maintenance policy as Nash equilibrium. The cooperative game, solved as a pure bargaining problem, defined as optimal solution the manufacturer's strategy of improved level of reliability for UAS components and user's strategy of UAS component replacement maintenance policy. Using the quantum based decision making model, the user's strategy of UAS component replacement maintenance policy is the most preferable; but the manufacturer's most preferable strategy depends on the biases, feelings captured with the criteria selected for determining attraction factor, as discussed above. Thus, it is shown in practice that these biases and feelings are indeed crucial in determining the optimal strategy for the stakeholder.

\section{Discussion and Conclusions}

The main objective of this research is to develop a decision making model employed in engineering design and any other social interplay between individuals, considered as stakeholders with different interests/stakes, capturing the complexity of human decision making under uncertainty. The decision makers' biases, emotions and subjective feelings apart from rational behaviour are incorporated in the proposed quantum-based decision making model.

Classical decision theory cannot explain human decision making because humans often violate the hypotheses of expected utility theory and make irrational choices. Quantum Decision theory is based on the similarity between mental processes and quantum mechanical phenomena with human decision making following the probabilistic behaviour of quantum mechanics. With the application of the quantum theory mathematics in decision making, the decision maker's mind is modelled as a quantum system of all superpositioned distinguished prospects/states. In QDT, human brain is assumed to have a dual nature and brain processes are divided into conscious and subconscious constituent parts. In the quantum-based decision making engineering design model, quantum techniques address entangled decision making by assessing intention interference. Hence, given a priori the preferences of the decision makers, this model can be utilised as an operational tool for decision making under uncertainty in the presence of entanglement assessing the quantum probabilities of the players' strategies in engineering design.

In the general application of QDT no prior information is assumed concerning the actions chosen by the players, (Yukalov and Sornette 2014), (Yukalov and Sornette 2014). Therefore, the expected values of the probabilities of the players' strategies are set to $1 / 2$ (non-informative prior) to compute the prospects' expected utilities, the utility factors and quantum probabilities. In the quantum-based decision making model these probabilities are initially set to $1 / 2$ as in QDT, however the engineering designer uses the computed values of the quantum strategic probabilities as available 
information to update the expected values of all probabilities of the players selecting their strategies. Hence, in the iteration formed, the values of the strategies' expected utility functions, the utility factors of equations (18) and the strategic probabilities of equations (19) are recalculated, until convergence is achieved between the values of strategies' probabilities and the quantum strategic probabilities of the players. The final converged values reflect the most accurate assessment of the quantum strategic probabilities.

As discussed in Section 0, three different attraction criteria were introduced to capture the biases, feelings and emotions of the stakeholders. The player's risk aversion and the associated attraction to the strategy offering the largest possible guaranteed gain is captured with the maximin criterion. The tendency towards reciprocal or conditional cooperation captures the feelings of mutual trust and reciprocity, and the strategies' attractiveness is assessed with the cooperation criterion, measured with the product of utility values of the players for all combinations of strategies. The players' attraction towards the strategy that offers maximal gain while at the same time minimizing the risk of minimal gain is captured with the attraction ratio criterion.

It is also possible to model players subconsciously governed by multiple biases, feelings and emotions. The appropriate aforementioned criteria will superimpose those feelings, emotions and biases, enforcing or cancelling out their individual effect. For example, as presented in Table 2, all three criteria were selected for a player driven by all corresponding feelings and emotions, to obtain an overall criterion used in the sign assignment of the attraction factor. In this case, for the engineering design game of Table 3, the original reliability strategy is less attractive to the manufacturer than the improved reliability strategy and the UAS component replacement strategy is more attractive to the user than the UAS replacement strategy. Alternatively, the cooperation and maximin criteria were selected to superimpose the player's attractions towards reciprocity, mutual trust and risk aversion. Another option is to select different criteria for each player, i.e. for one player attracted to reciprocity and mutual trust use the cooperation criterion, while for the other risk averse player select the maximin criterion. For the specific engineering design game, the UAS component replacement policy was found more attractive with all possible combinations of attraction criteria. Other feelings, emotions and biases of the stakeholders could be included in the sign assignment of the attraction factors by adding appropriate attraction criteria. For instance, a manufacturer could be biased towards a specific reliability level strategy, because of previously supplied components that would have to be replaced by new ones in a change of strategy; an additional attraction criterion could model this bias.

Concerning the interference/attraction factors used in equations (19), Yukalov and Sornette (2011) assume no prior information and assign a value of $1 / 2$ to the uncertainty factors and the expected values of the probabilities in the square roots of equation (16) to derive the quarter law for the attraction factor. In general, the attraction factors are subjective quantities, which are not only different for different decision makers, but they are also different for the same decision maker for different prospects/strategies and even different for the same decision maker and prospects at different times. They are always obtained from equations (16) and based on Theorem 1 of (Yukalov and Sornette 2011) they have the property of interference alteration (i.e. their sum is zero): $\sum_{j=1}^{N} q\left(\pi_{j}\right)=0$. To get a better than the quarter law estimate of the interference term of the players' strategies in the proposed quantum-based decision making model, the values of joint probabilities $p\left(S_{1} S_{1}{ }^{\prime}\right), p\left(S_{1} S_{2}{ }^{\prime}\right), p\left(S_{2} S_{1}{ }^{\prime}\right)$ and $p\left(S_{2} S_{2}{ }^{\prime}\right)$ were computed from the conditional probabilities since: $p\left(S_{i} S_{j}{ }^{\prime}\right)=p\left(S_{i} \mid S_{j}{ }^{\prime}\right) p\left(S_{j}\right)$. They were then employed in equation (16) to get more accurate estimates of the attraction factors:

$$
q\left(S_{1}\right)=2 \varphi\left(S_{1}\right) \sqrt{p\left(S_{1} S_{1}{ }^{\prime}\right) p\left(S_{1} S_{2}{ }^{\prime}\right)}, q\left(S_{2}\right)=2 \varphi\left(S_{2}\right) \sqrt{p\left(S_{2} S_{1}{ }^{\prime}\right) p\left(S_{2} S_{2}{ }^{\prime}\right)}
$$

The key findings/contributions of the proposed method can be summarized as follows:

- The quantum-based engineering design model is an operational tool for decision making under uncertainty, assessing the quantum probabilities of the strategies in engineering design based on both the rationality and the biases, feelings and emotions of the stakeholders involved.

- Attraction criteria are introduced to capture different biases, feelings and emotions. Moreover, multiple biases, feelings and emotions can be superimposed, cancelling or enforcing their individual effect, by selecting more than one criterion.

- The attraction factors are not assigned a constant value of $1 / 4$, but instead the values of the quantum strategic probabilities and attraction factors of the players are updated and more accurately assessed, following the process of Figure 2. 
The conditional probabilities can also be assessed based on data from experimental realization with the actual subjects choosing from the specific prospects at certain times, given that they know their opponents' selection, as in (Tversky and Shafir 1992). Hence, further similar experiments can be done to assess these conditional probabilities and obtain more accurate estimates of the interference terms $q(\pi)$ for the specific subjects and chosen strategies. These experiments are required to not only validate the estimates of the attraction factors but also validate the proposed model based on the selections of decision makers involved in the engineering design used in the assessment of the aforementioned probabilities. Nevertheless, if no other information is available, this model provides a first estimate of the probabilities of the players' strategies.

\section{References}

Agrawal, P. M., \& Shard, R. (2013). OR Forum - Quantum Mechanics and Human Decision Making. Operations Research, 61(1), 1-16. https://doi.org/10.1287/opre.1120.1068

Allais, M. (1953). Le Comportement de l' Homme Rationnel Devant le Risque: Critique de Postulats et Axiomes de l' Ecole Americaine. Econometrica, 21, 503-546. https://doi.org/10.2307/1907921

Arsenyan, J., Buyukozkan, G., \& Feyzioglu, O. (2015). Modeling Collaboration Formation With a Game Theory Approach. Expert Systems with Applications, 42, 2073-2085. https://doi.org/10.1016/j.eswa.2014.10.010

Ashtiani, M., \& Azgomi, M. A. (2015). A Survey of Quantum-like Approaches to Decision Making and Cognition. Mathematical Social Sciences, 75, 49-80. https://doi.org/10.1016/j.mathsocsci.2015.02.004

Bruza, P., Sofge, D., Lawless, W., Van Rijsbergen, K., \& Klusch, M. (2009). Quantum Interaction. Third International Symposium, QI 2009, Saarbrucken, Germany. https://doi.org/10.1007/978-3-642-00834-4

Busemeyer, J. R. (2009). Introduction to quantum probability for social and behavioral scientists. Quantum Interaction 3rd International Symposium, QI 2009 Proceedings. https://doi.org/10.1007/978-3-642-00834-4_1

Busemeyer, J. R., Wang, Z., \& Townsend, J. T. (2006). Quantum Dynamics of Human Decision-making. Journal of Mathematical Psychology, 50, 220-241. https://doi.org/10.1016/j.jmp.2006.01.003

Desideri, J. A. (2012). Cooperation and Competition in Multidisciplinary Optimization. Comput. Optim. Appl., 52, 29-68. https://doi.org/10.1007/s10589-011-9395-1

Dixit, V. V., \& Denant-Boemont, L. (2014). Is Equilibrium in Trasport Pure Nash, Mixed or Stochastic? Transportation Research: Part C, 48, 10. https://doi.org/10.1016/j.trc.2014.09.002

Ellsberg, D. (1990). Risk, Ambiguity and the Savage Axioms. New York, NY, USA, Cambridge University Press.

Fishburn, P. C. (1970). Utility Theory for Decision Making. New York, John Wiley \& Sons.

Hargreaves, H. S. P., \& Varoufakis, Y. (2004). Game Theory: A Critical Text. London \& New York, Routledge.

Harsanyi, J. C. (1995). A New Theory of Equilibrium Selection for Games with Complete Information. Games and Economic Behavior, 8, 91-122. https://doi.org/10.1016/S0899-8256(05)80018-1

Harsanyi, J. C., \& Selten, R. (1972). A Generalized Nash Solution for Two-person Bargaining Games with Incomplete Information. Management Science, 18(5). https://doi.org/10.1287/mnsc.18.5.80

He, F., Yin, Y., \& Shirmohammadi, N. (2013). Tradable Credit Schemes on Networks with Mixed Equilibrium Behaviours. Transportation Research: Part B, 57, 47-65. https://doi.org/10.1016/j.trb.2013.08.016

Jammer, M. (1966). The Conceptual Development of Quantum Mechanics, McGraw-Hill.

Jiao, Y., Ma, M., Yu, Q., Yi, K., \& Ma, Y. (2011). Quality of Service Provisioning in Worldwide Interoperability for Microwave Access Networks Based on Cooperative Game Theory. IET Commun, 5(3), 284-295. https://doi.org/10.1049/iet-com.2010.0304

Keeney, R. L., \& Raiffa, H. (1976). Decisions with Multiple Objectives. Cambridge, UK, Press Syndicate of the Cambridge University.

Khrennikov, A. (2010). Ubiquitous Quantum Structure: From Psychology to Finance, Heidelberg and New York: Springer. https://doi.org/10.1007/978-3-642-05101-2

Ledyard, J. O. (1995). Public Goods: a Survey of Experimental Research, in J.H. Kagel, A.E. Roth (Eds.), The Handbook of Experimental Economics. Princeton, NJ, USA, Princeton University Press.

Nash, J. F. (1950). The Bargaining Problem. Econometrica, 18(2), 155-162. https://doi.org/10.2307/1907266

Nash, J. F. (1950). Non Co-operative Games. PhD, Princeton. 
Papageorgiou, E., Eres, H., \& Scanlan, J. (2015). Value Driven Conceptual Design of Unmanned Air System for Defense Applications. AIAA Aviation Conference 2015. Dallas, TX, USA. https://doi.org/10.2514/6.2015-2736

Papageorgiou, E., Eres, H., \& Scanlan, J. (2016). Value Modelling for Multi-Stakeholder and Multi-Objective Optimization in Engineering Design. Journal of Engineering Design. Accepted for Publication. https://doi.org/10.1080/09544828.2016.1214693

Savage, L. J. (1954). The foundations of Statistics, New York: Wiley.

Semsar-Kazerooni, E., \& Khorasani, K. (2009). Multi-agent Team Cooperation: a Game Theory Approach. Automatica 45, 2205-2213. https://doi.org/10.1016/j.automatica.2009.06.006

Trappey, A., Trappey, C., \& Ni, W. C. (2013). A Multi-Agent Collaborative Maintenance Platform Applying Game Theory Negotiation Strategies. J. Intell. Manuf., 24, 613-623. https://doi.org/10.1007/s10845-011-0606-5

Tversky, A., \& Kahneman, D. (1981). The Framing of Decisions and the Psychology of Choice. Science, New Series 211(4481), 453-458.

Tversky, A., \& Kahneman, D. (1983). Extensional Versus Intuitive Reasoning: the Conjunction Fallacy in Probability Judgment. Psychol. Rev., 90, 293-315. https://doi.org/10.1037/0033-295X.90.4.293

Tversky, A., \& Kahneman, D. (1986). Rational Choice and the Framing of Decisions. Journal of Business, 59(4), 29. https://doi.org/10.1086/296365

Tversky, A., \& Shafir, E. (1992). The Disjunction Effect in Choice Under Uncertainty. Psychological Science 3, 305-309. https://doi.org/10.1111/j.1467-9280.1992.tb00678.x

Von Neumann, J., \& Morgenstern, O. (1953). Theory of Games and Economic Behavior. Princeton,NJ, Princeton University Press.

Xiao, A., Zeng, S., Allen, J. K., Rosen, D. W., \& Mistree, F. (2005). Collaborative Multidisciplinary Decision Making Using Game Theory and Design Capability Indices. Research in Engineering Design, 16, 57-72. https://doi.org/10.1007/s00163-005-0007-x

Yukalov, V. I., \& Sornette, D. (2011). Decision Theory with Prospect Interference and Entanglement. Theory and Decision, 70, 283-328. https://doi.org/10.1007/s11238-010-9202-y

Yukalov, V. I., \& Sornette, D. (2014). How Brains Make Decisions. Springer Proc. Phys., 150, 37-53. https://doi.org/10.1007/978-3-319-02207-9_11

Yukalov, V. I., \& Sornette, D. (2014). Manipulating Decision Making of Typical Agents. IEEE Transactions on Systems, Man and Cybernetics, 44(9), 14. https://doi.org/10.1109/tsmc.2014.2314283

Yukalov, V. I., \& Sornette, D. (2015). Quantum Probability and Quantum Decision-making. Phil. Trans. R. Soc. A, 374, 15. https://doi.org/10.1098/rsta.2015.0100

Yukalov, V. I., \& Sornette, D. (2015). Role of Information in Decision Making of Social Agents. Int. J. Inf. Technol. Decis. Mak., 14, 1129-1166. https://doi.org/10.1142/S0219622014500564

Zhong, W., Xu, Y. Y., Wang, J. H., Li, D. P., \& Tianfield, H. (2014). Adaptive Mechanism Design and Game Theoretic Analysis of Auction-driven Dynamic Spectrum Access in Cognitive Radio Networks. EURASIP Journal on Wireless Communications and Networking, 14. https://doi.org/10.1186/1687-1499-2014-44

\section{Copyrights}

Copyright for this article is retained by the author(s), with first publication rights granted to the journal.

This is an open-access article distributed under the terms and conditions of the Creative Commons Attribution license which permits unrestricted use, distribution, and reproduction in any medium, provided the original work is properly cited. 